\title{
De I'idée au marché : I'ITERG renforce ses activités dans la chimie verte avec la plateforme CEDOP, Centre européen de développement des oléo-produits
}

\author{
Carine ALFOS \\ Xavier PAGĖS-XATART-PARÈS \\ ITERG, \\ Institut des Corps Gras, \\ 11 rue Gaspard Monge, \\ 33600 Pessac, \\ France \\ $<$ c.alfos@iterg.com>
}

\begin{abstract}
ITERG, the Industrial Technical Centre (CTI) for Fats and Oils, under the supervision of the Ministry of Economy, Finance and Employment, located in Pessac has undertaken the project to build a technology platform (to be operational in early 2012) dedicated to innovation and transfer in the field of oleochemicals from experimental to semi-industrial scales. This platform, CEDOP (European Centre for the Development of Oleo-Products) can offer within the same entity, expertise and scalability from laboratory to semi-industrial productions, to industrial manufacturers, processors, distributors and value chain actors of oleo-products. This project unique in its design, perfectly in tune with public policies for innovation and development of green chemistry, not only meets the needs of manufacturers and their markets but also the current socio-economic expectations in terms of environment and sustainable development. The CEDOP project total investment reached 8,700,000 $€$ [buildings (laboratories, industrial and pilot halls, technical offices) and equipment]. After the positive results of preliminary studies (competition, market research, risk assessment) and the inclusion of CEDOP in the "State-Aquitaine Region Contract of Projects", public institutions (Regional Council, State, FEDER, local) and professional organization (ONIDOL) brought together $85 \%$ of the project funding. Such a support reflects the confidence of the stakeholders in ITERG's independent entrepreneurial skills in scientific and technology to meet public and professional policies, but above all in the large scope of corporate customers from different sectors of activity, potential future users of the tool. This project for research and technology transfer is fully integrated in ITERG's development and diversification strategies and aims to develop an attractive lipochemistry-technology cluster.
\end{abstract}

Key words: oleo-products development platform, pilot plants, semi-industrial scale production, fats \& oils derivatives

et acteurs des filières des oléo-produits. Ce projet unique dans sa conception, parfaitement en phase avec les politiques publiques en matière d'innovation et de développement de la chimie verte, répond non seulement aux besoins des industriels et de leurs marchés mais aussi aux attentes socio-économiques actuelles en matière d'environnement et de développement durable.

\section{Histoire du projet CEDOP}

Le montant de l'investissement du projet CEDOP s'élève à $8700000 €$ [bâtiments (laboratoires, hall industriel, hall technique, bureaux) et équipements]. Après les résultats positifs des études préliminaires (analyse de la concurrence, étude de marchés au niveau européen, évaluation des risques, business plan à 7 ans) et l'inscription du projet CEDOP au Contrat de projets État-région Aquitaine, les bailleurs publics (Conseil Régional, État, FEDER, collectivités locales) et professionnels (ONIDOL) sollicités ont contribué pour $85 \%$ du financement nécessaire. L'importance de cette contribution traduit la confiance des acteurs dans le savoir-faire scientifique et tech-

Pour citer cet article : Alfos $C$, Pagès-Xatart-Parès X. De l'idée au marché : I'ITERG renforce ses activités dans la chimie verte avec la plateforme CEDOP, Centre européen de développement des oléo-produits. OCL 2012 ; 19(1) : 54-58. doi : 10.1684/ocl.2012.0430 


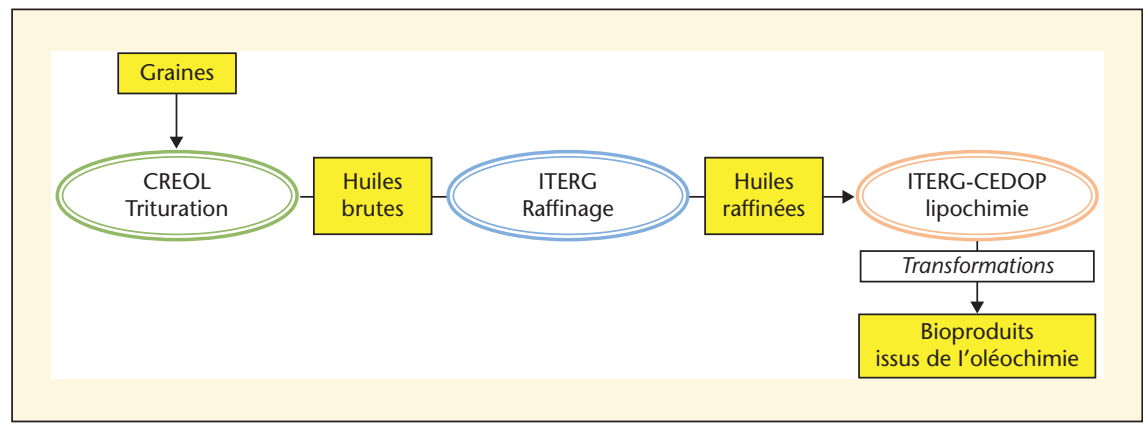

Figure 1. Plateformes semi-industrielles du site de Pessac. Toutes les opérations peuvent être réalisées sur des quantités allant de l'échelle laboratoire $(<1 \mathrm{~kg})$ jusqu'à l'échelle de la tonne en passant par le stade pilote (de 2 à $100 \mathrm{~kg}$ ). Le site offre des outils unitaires au service d'une filière intégrée dont les partenaires occupent à la fois l'amont et l'aval de la production. Ainsi, des solutions globales peuvent être apportées à des problématiques allant de la production de graines à la valorisation des produits finis, en incluant la transformation. Dans ce cadre, les différentes unités prennent part à des actions concertées, tant nationales qu'européennes, dans les domaines de la valorisation des huiles ou des protéines, pour des usages alimentaires ou industriels.

nologique et l'esprit d'entreprise indépendant de I'ITERG mis au service des politiques publiques et professionnelles mais aussi et surtout, dans le vaste éventail d'entreprises clientes issues de secteurs d'activités très diversifiés et futures utilisatrices de l'outil.

Ce projet technologique de recherche et de transfert s'inscrit pleinement dans la stratégie de développement et de

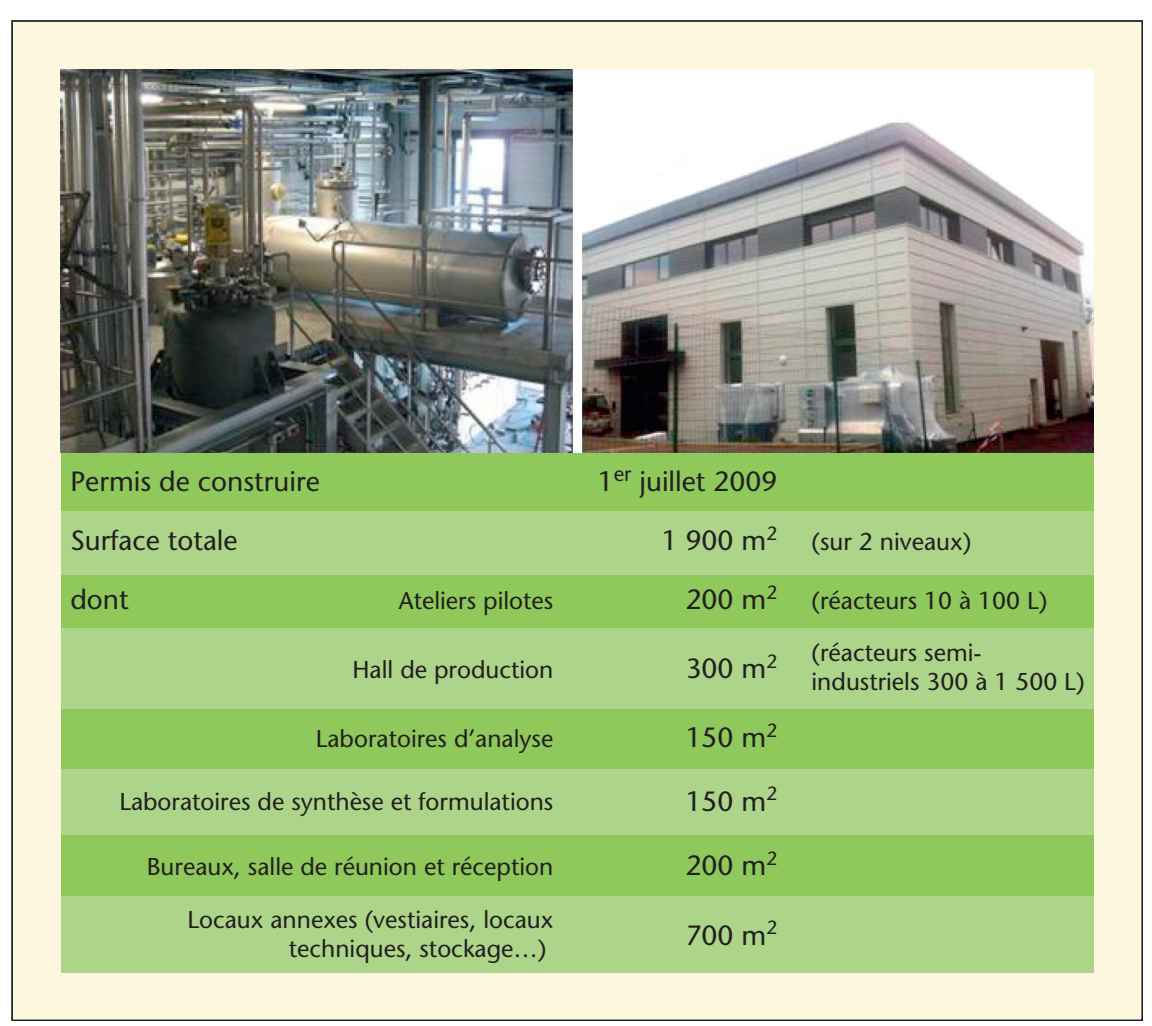

Figure 2. Les caractéristiques de superficies du nouveau bâtiment du CEDOP. d'intégrer les problématiques et contraintes industrielles (optimisation des procédés, bilans économiques, bilan environnemental) ; jusqu'à ce jour, les produits mis au point à l'échelle du laboratoire étaient directement développés par les industriels avec les incertitudes, le coût et les contraintes que représente l'absence d'une étape intermédiaire préindustrielle.

La plateforme CEDOP spécialisée dans le développement à vocation industrielle (hall industriel équipé de réacteurs de $200 \mathrm{~kg}$ à 1 tonne de capacité) de bioproduits issus de l'oléochimie permettra ainsi aux industriels d'optimiser et d'accélérer la mise sur le marché de ces nouveaux produits tout en limitant les risques et les coûts associés. De plus, I'outil CEDOP pourra aussi répondre à des demandes de production de faible tonnage pour des marchés de niche.

La plateforme CEDOP s'intègre parfaitement dans une logique de synergie et de bioraffinerie préindustrielle avec les outils et les moyens techniques existants du site de Pessac (figure 1), à savoir : le CREOL, Centre de recherche et d'expérimentation sur les oLéagineux et les protéagineux (filiale du CETIOM Centre technique interprofessionnel des oléagineux métropolitains - et de SOFIPROTEOL, acteur financier et industriel de la filière française des huiles et protéines végétales) et l'unité de raffinage ITERG, installation permettant tout ou partie des opérations unitaires du raffinage chimique ou physique des huiles et des corps gras. Dans un environnement technique performant, cette unité de raffinage offre aux industriels un site privilégié pour produire des corps gras raffinés spécifiques avec une totale garantie de traçabilité et de qualité.

\section{Description de la plateforme CEDOP}

La figure 2 présente les caractéristiques de superficies du nouveau bâtiment et la figure 3 détaille les équipements disponibles en rappelant ceux des deux autres unités créant toute la cohérence du site rappelée sur la figure 1, chaque plateforme offrant les deux niveaux de fabrication pilote pour essais et industriels pour préséries. 


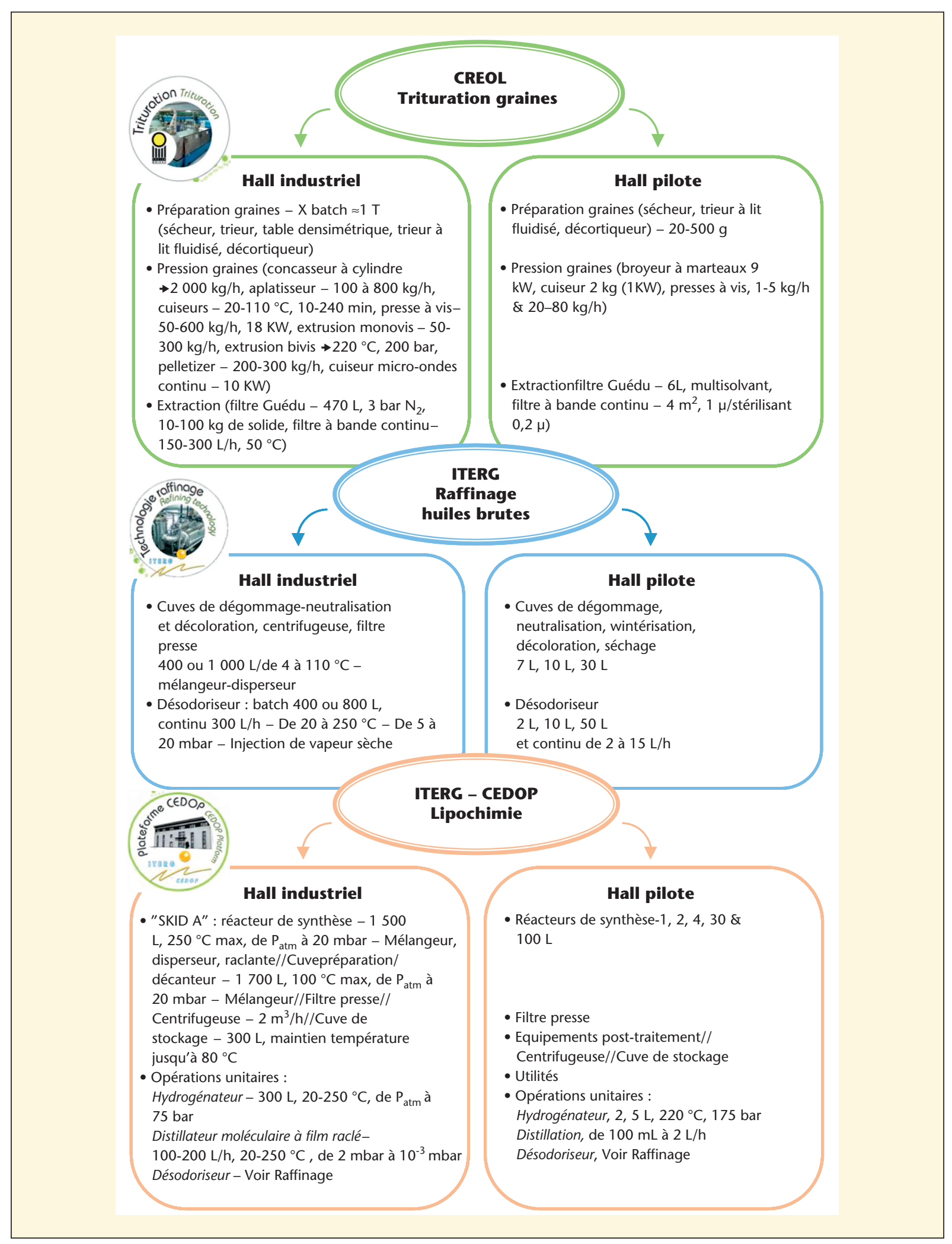

Figure 3. Les équipements disponibles sur la plateforme CEDOP. 


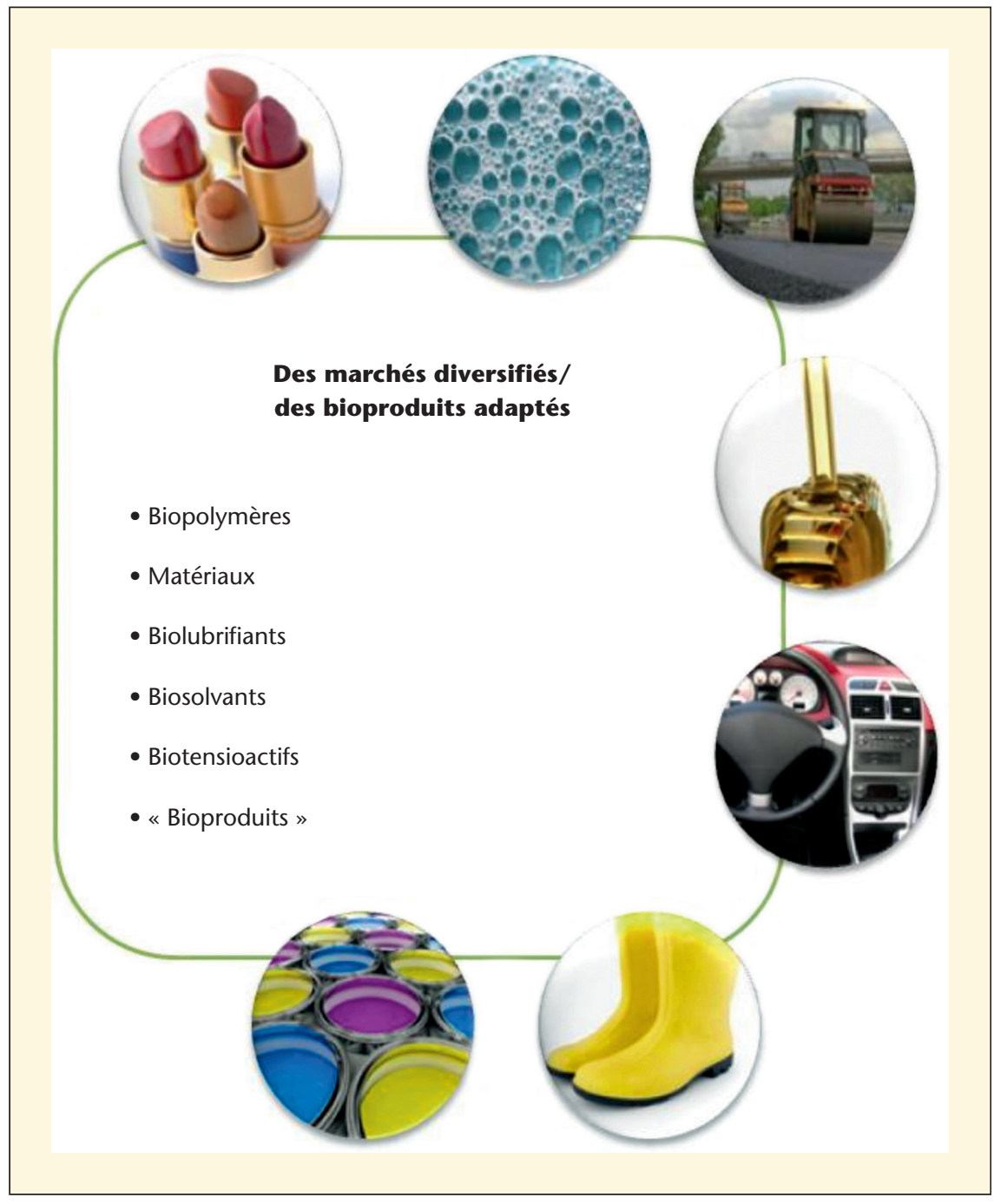

Figure 4. Les secteurs industriels sont très variés.

\section{Projets, applications et innovations produits}

Le projet CEDOP constitue par son ampleur un enjeu majeur du développement et, à ce titre, doit permettre de franchir un cap décisif en matière de périmètre d'activité, de rayonnement technologique et d'attractivité du territoire.

À ce jour, les nombreuses démarches entreprises depuis le lancement du projet pour promouvoir et faire connaître la plateforme CEDOP auprès du monde de la recherche et de l'industrie, ont permis d'initier des projets de R\&D et d'innovation. Parmi les projets lancés en 2011 qui n'auraient pas pu voir le
Par ailleurs, une quinzaine de contrats industriels mobilisant CEDOP ont été signés, impliquant des $\mathrm{PME}$ et des grands groupes d'envergure mondiale.

II faut aussi souligner que CEDOP a été I'élément-clé qui a permis à I'ITERG de se positionner comme partenaire du projet d'Institut d'Excellence dans le domaine des énergies décarbonnées PIVERT (Picardie innovations végétales enseignements et recherches technologiques) lancé dans le cadre des appels d'offre Investissements d'Avenir. Ce projet doté d'un budget de 220 millions d'euros sur dix ans, confère à l'ITERG et à CEDOP une dimension nationale, reconnue par les pouvoirs publics. II permettra aux entreprises régionales et nationales un accès facilité aux 150 chercheurs, ingénieurs et enseignants travaillant dans les différents laboratoires et aux pilotes industriels impliqués, dans les domaines de la transformation de la biomasse oléagineuse, contribuant ainsi à assurer le transfert des innovations du laboratoire au stade préindustriel.

Plus récemment, dans le cadre des Investissements d'Avenir, I'ADEME a lancé un appel à manifestation d'intérêt sur l'action "démonstrateurs et plateformes technologiques en énergies décarbonées et chimie verte". Sur cet appel d'offres, I'ITERG a participé au dépôt de deux projets d'envergure, pour lesquels la plateforme CEDOP est fortement mobilisée.

D'autre part, dans le cadre de l'institut Carnot LISA dont I'ITERG est I'un des membres fondateurs, la mise en œuvre de CEDOP a été clairement affichée sur I'axe chimie verte du plan stratégique de développement de I'Institut.

Les applications attendues concernent la synthèse ou la purification d'acides gras et esters d'acides gras, d'amines et de bétaïnes, de dimères d'acides gras, de produits hydrogénés, d'esters $d$ 'huiles rares, d'huiles soufflées, de standolies, d'huiles interestérifiées, de glycolipides, d'amides grasses, de savons (liquides, secs), d'esters de polysaccharides, et de manière générale de nouvelles biomolécules. Celles-ci obtenues et produites en pré-séries sur la plateforme CEDOP peuvent être des synthons, "pré-polymères ", à combiner entre eux ou avec d'autres (par exemple, ligno-cellulosiques) pour obtenir des polymères biosourcés avec des procédés propres 


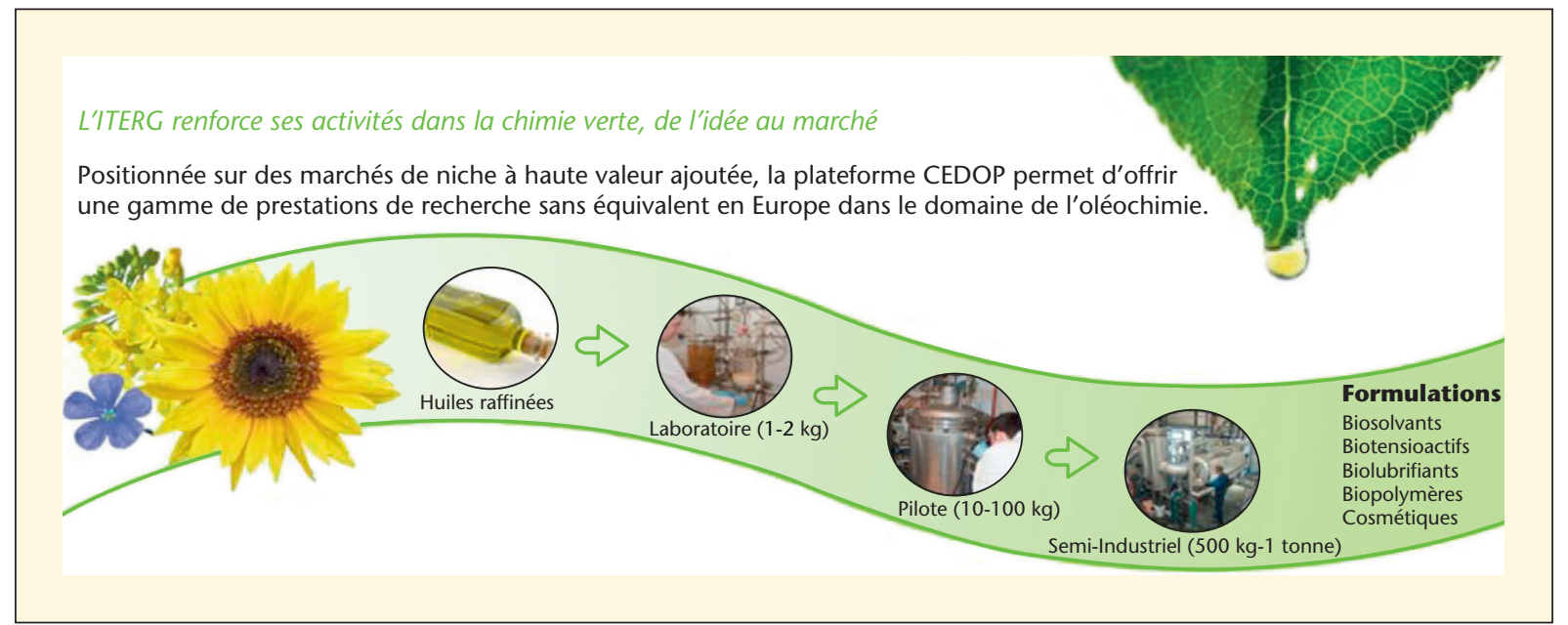

Figure 5. L'ITERG renforce ses activités dans la chimie verte, de l'idée au marché.

faisant appel à des catalyseurs organiques ou aux biotechnologies.

Les secteurs industriels sont très variés et vont de l'alimentaire aux polymères biosourcés, en passant par les produits cosmétiques, les détergents, les peintures, encres et vernis, les revêtements, les biolubrifiants, sans oublier les biocarburants (figure 4). L'ITERG a apporté ses compétences à l'étude des biodiesels. Sa contribution sera dorénavant recherchée sur la mise au point des biocarburants de troisième génération.

Le développement du portefeuille de brevets sera poursuivi dans le cadre de la politique partenariale. Fort de ses liens avec I'Université, I'ITERG pourra également s'appuyer sur la SATT (Sociétés d'accélération du transfert de technologie) de Bordeaux pour valoriser ses brevets obtenus en copropriété avec ses partenaires académiques, dans un univers devenu très concurrentiel. Les relations privilégiées au sein du Réseau CTI (Centre technique industriel) et les liens des autres centres techniques avec de nombreuses branches industrielles seront sollicités pour identifier de nouvelles applications sectorielles de ses brevets génériques. Dans le cadre de contrats privés, soutenus ou non par les dispositifs d'aides de l'État, des brevets d'applications pourront être concédés ou déposés en partenariat avec les entreprises. L'ITERG envisage de réinvestir les retours financiers de ses brevets dans les équipements nécessaires à l'activité d'intérêt collectif.

\section{Conclusion}

L'ensemble de ces éléments témoigne de l'attractivité de CEDOP vis-à-vis des industriels et du monde académique, participe à la lisibilité technique et scientifique de I'ITERG et positionne la nouvelle plateforme comme l'un des acteurs majeurs au niveau national dans le domaine de la chimie verte. L'ITERG réalise différents types de prestations de $R$ $\& D$ privées auprès d'industriels (PME et grands groupes). Celles-ci vont du projet d'innovation de courte durée aux projets pluriannuels associant en général des grands comptes industriels. Naturelle- ment, plus la recherche est proche du marché (recherche industrielle, a fortiori développement expérimental), plus le caractère économique de la prestation s'impose, avec exclusivité et transfert complet de la valeur créée (propriété des résultats).

La possibilité de mener des projets "de l'idée au marché " (figure 5) est rendue possible sur un seul et même site (Pessac/ Bordeaux) concentrant les équipes de recherche (laboratoires), la mise au point des procédés à l'échelle pilote (de 1 à 50$100 \mathrm{~kg}$ ), scale-up avec le hall industriel CEDOP (quelques tonnes) et/ou l'unité de raffinage.

La plateforme technologique CEDOP étant dorénavant partie prenante de I'IEED PIVERT, une dynamique privilégiée depuis la recherche académique (cofinancement de thèse) jusqu'au transfert sur équipements semi-industriels apportera une ligne directrice forte aux travaux de recherche, avec un gradient croissant de participation industrielle depuis la recherche amont jusqu'à la production de préséries industrielles. 